\title{
A DEFINIÇÃO DE POTENCIAL QUÍMICO EM FONTES
} DIDÁTICAS

\author{
THE CHEMICAL POTENTIAL DEFINITION IN DIDACTIC SOURCES
}

\section{LA DEFINICIÓN DE POTENCIAL QUÍMICO EN FUENTES DIDÁCTICAS}

DOI: $10.22481 / \mathrm{rbba} . v 10 \mathrm{i} 01.8003$

\begin{abstract}
Jornandes Jesús Correia
Universidade Estadual do Sudoeste da Bahia, Bahia, Brasil

Lattes iD: http://lattes.cnpq.br/5594644574555987

Orcid: https://orcid.org/0000-0002-0035-2300

Endereço eletrônico: jornandes.correia@uesb.edu.br

Gabriel Fonseca Guimarães Universidade Estadual do Sudoeste da Bahia, Bahia, Brasil

ORCID: https://orcid.org/0000-0002-8795-897X

ID Lattes: http://lattes.cnpq.br/6850946554302469

Endereço eletrônico: gabrielfonseca1415@gmail.com
\end{abstract}

\begin{abstract}
RESUMO
Este artigo tem por objetivo apresentar as análises das abordagens sobre o Potencial Químico em livros de texto, em dicionários, em enciclopédias e em sites da Internet, bem como investigar a apresentação da definição, da conservação, dos seus valores máximo e mínimo e da variação da sua intensidade. A Categoria de Análise das definições está referenciada na abordagem histórica e no Silogismo de Aristóteles. Observou-se que algumas fontes pesquisadas estabeleceram como premissa a relação existente entre o Potencial Químico e a Energia Química para comparar com a relação existente entre a Temperatura
\end{abstract}


e a Energia Térmica. Observou-se também a superficialidade nas abordagens e a prioridade a um tratamento matematizado para associar Potencial Químico a Equilíbrio Químico, em detrimento de uma abordagem epistemológica ou história. Devido à falta de sintonia das definições apresentadas nas fontes pesquisadas, vimos apresentar uma definição para o Potencial Químico. Embora Potencial Químico não seja uma grandeza potencial propriamente dita, a exemplo da Energia Interna, da Entalpia, da Entropia, do Potencial de Helmholtz e do Potencial de Gibbs, entendemos que o definir ainda constitui um desafio. Como perspectiva futura, este grupo de pesquisa visa divulgar as conclusões de suas pesquisas sobre as definições em fontes didáticas dos potenciais termodinâmicos para sistemas abertos.

Palavras-chave: Potencial Químico; Equilíbrio Químico; Funções Potenciais da Termodinâmica; Ensino da Termodinâmica.

\section{RESUMEN}

Este artículo tiene como objetivo presentar análisis de aproximaciones al Potencial Químico en libros de texto, diccionarios, enciclopedias y sitios de Internet, así como investigar la presentación de la definición, conservación, sus valores máximos y mínimos y la variación de su intensidad. La categoría de análisis de definiciones se hace referencia en el enfoque histórico y el silogismo de Aristóteles. Se observó que algunas fuentes investigadas han establecido como premisa la relación entre Potencial Químico y Energía Química para comparar con la relación entre Temperatura y Energía Térmica. También se observó la superficialidad en los enfoques y la prioridad a un tratamiento matemático para asociar el Potencial Químico al Equilibrio Químico, en detrimento de un enfoque epistemológico o histórico. Debido a la falta de armonía entre las definiciones presentadas en las fuentes investigadas, hemos llegado a una definición de Potencial químico. Aunque el Potencial químico no es una cantidad potencial per se, como Energía Interna, Entalpía, Entropía, Potencial de Helmholtz y Potencial de Gibbs, entendemos que definirlo sigue siendo un desafío. Como perspectiva de futuro, este grupo de investigación tiene como objetivo difundir las conclusiones de su investigación sobre las definiciones en fuentes didácticas de los potenciales termodinámicos para sistemas abiertos. 
Palabras clave: Potencial químico; Equilibrio químico; Funciones potenciales de la termodinámica; Enseñanza de la termodinámica.

\begin{abstract}
This article aims to present analyzes of approaches to Chemical Potential in textbooks, dictionaries, encyclopedias and Internet sites, as well as to investigate the presentation of definition, conservation, its maximum and minimum values and variation of the his intensity. The Analysis Category of definitions is referenced in Aristotle's historical approach and syllogism. It was observed that some researched sources have established as a premise the relationship between Chemical Potential and Chemical Energy to compare with the relationship between Temperature and Thermal Energy. It was also observed the superficiality in the approaches and the priority to a mathematic treatment to associate Chemical Potential to Chemical Equilibrium, in detriment of an epistemological approach or history. Due to the lack of harmony between the definitions presented in the researched sources, we have come up with a definition for the Chemical Potential. Although Chemical Potential is not a potential quantity per se, like Internal Energy, Enthalpy, Entropy, Helmholtz Potential and Gibbs Potential, we understand that defining it is still a challenge. As a future perspective, this research group aims to disseminate the conclusions of its research on the definitions in didactic sources of the thermodynamic potentials for open systems.
\end{abstract}

Keywords: Chemical Potential; Chemical Equilibrium; Potential Functions of Thermodynamics; Teaching of Thermodynamics.

\title{
1 INTRODUÇÃO
}

Potencial Químico é uma grandeza do conteúdo tanto da Termodinâmica do Equilíbrio quanto da Química, mais precisamente no tópico que aborda as reações químicas (BRASIL) da Físico-química. A necessidade de dominar a significação de Potencial Químico se deve à sua importância no controle de uma reação química, bem como numa análise de transição de fase, de difusão de átomos e no equilíbrio químico de um sistema termodinâmico.

Definições de grandezas de suma importância, que estão estruturadas com superficialidade, com imprecisão e sem a devida clareza, podem dificultar a compreensão, 


\section{A DEFINIÇÃO DE POTENCIAL QUÍMICO EM FONTES DIDÁTICAS}

sobretudo das grandezas físicas que compõem o escopo da Termodinâmica (CORREIA; GUIMARÃES, 2020). Nessa perspectiva, Porto e Silva (2019) afirmam que as grandezas da Físico-Química estão definidas de forma abstrata. Tsaparlis e Finlayson (2013, p. 257) chamam a atenção sobre a importância dessa área de conhecimento, advertindo que "a Físico-Química é considerada um curso essencial na educação e no treinamento, não apenas dos químicos, mas também de muitos outros especialistas".

Quanto ao Potencial Químico, Porto e Silva (2019, p. 112) fizeram uma análise histórica das abordagens dos livros didáticos e concluíram:

Nossa experiência com o estudo e o ensino da Termodinâmica tem revelado que o conceito de potencial químico - extremamente importante no desenvolvimento da Termodinâmica do equilíbrio químico — apresenta uma dificuldade ímpar para os estudantes dos cursos de Química.

Essa falta de clareza na definição do Potencial Químico é recorrente para diversas grandezas nas mais variadas áreas de conhecimento. Essa hipótese vem sendo confirmada pelos resultados das ações de uma das linhas de pesquisa do Grupo de Estudos e Pesquisas em Didática das Ciências Experimentais e da Matemática (GDICEM), vinculado ao Museu Pedagógico Casa Padre Palmeira (MP) e confirmada pelas investigações de definições em fontes didáticas tanto de Funções de Estado quanto de Funções de Transformação da Termodinâmica do Equilíbrio. Essa linha de pesquisa do GDICEM iniciou suas atividades em 2008 com a submissão do Projeto de Pesquisa intitulado "Os conceitos em livros didáticos de Física - problemas relacionados às definições de fenômenos físicos", junto ao Departamento de Ciências Exatas e Tecnológicas (DCET) e cadastrado junto à Gerência de Pesquisa (GP) da Pró-Reitoria de Pesquisa e Pós-Graduação (PPG) da Universidade Estadual do Sudoeste da Bahia (UESB), Campus de Vitória da Conquista, Bahia, Brasil. Vimos listar alguns resultados dessa pesquisa: Calor (CORREIA; MAGALHÃES; LIMA, 2008) e (CORREIA; MAGALHÃES, 2009); Trabalho (CORREIA; JOSÉ, 2011); Entropia (CORREIA; JOSÉ, 2013); Conservação de Energia (CORREIA; ORTIGOZA, 2015); Temperatura (CORREIA, 2017); Energia Interna (CORREIA; OLIVEIRA, 2018); Entalpia (CORREIA; OLIVEIRA, 2019); Energia Livre de Helmholtz (CORREIA; GUIMARÃES, 2020) e Energia Livre de Gibbs (CORREIA; GUIMARÃES, 2021, no prelo).

Dando continuidade às ações dessa linha que pesquisa, vimos analisar, em fontes didáticas, além das definições, as discussões da variação da intensidade do Potencial Químico, 
bem como da sua conservação e dos seus valores máximo e mínimo. Devido ao tratamento matematizado das discussões apresentadas pelas fontes analisadas, bem como pela falta de sintonia nas premissas adotadas, vimos propor uma definição para o Potencial Químico, referenciado no Silogismo de Aristóteles, de modo a contribuir para o ensino e para a aprendizagem tanto da Física quando da Química.

Como perspectiva futura, este grupo de pesquisa visa publicar as suas análises em fontes didáticas das definições dos grandes potenciais da termodinâmica.

\section{MATERIAIS E MÉTODOS}

Potencial Químico tem importância fundamental nas Reações Químicas. Logo, é uma das grandezas fundamentais para a Físico-Química, por estar inserido nas equações de estado que envolve as Funções Potenciais da Termodinâmica para sistemas abertos.

Considerando que o desequilíbrio termodinâmico é decorrente da diferença dos valores dos Potenciais Químicos entre sistemas em interação é que foi analisada a definição de Potencial Químico em fontes didáticas, cuja categoria de análise se baseia no Silogismo Aristotélico.

Aristóteles não se preocupou apenas com os problemas das ciências teóricas, mas também com as questões epistemológicas da pesquisa científica (NAKAZA; LINHARES, 2017, p. 2). Para Aristóteles, as definições e as demonstrações necessitam fortemente do artifício do silogismo científico para serem traduzidas em conhecimento, conforme está evidenciado na tradução de Angioni (2004, p. 15): “por 'demonstração' entendo silogismo científico; e por 'científico' entendo aquele segundo o qual conhecemos cientificamente por possuí-lo". Logo, a elaboração de definições e de demonstrações necessita de premissas verdadeiras, conforme Aristóteles (350 a. C., 71b 19):

Assim, se conhecer cientificamente é como propusemos, é necessário que o conhecimento demonstrativo provenha de itens verdadeiros, primeiros, imediatos, mais cognoscíveis que a conclusão, anteriores a ela e que sejam causas dela. Pois é deste modo que os princípios serão de fato apropriados ao que se prova. É possível haver silogismo mesmo sem tais itens, mas não é possível haver demonstração. Pois, tal silogismo não poderia propiciar conhecimento científico. (Apud ANGIONI, 2004, p. 15). 
Sob a perspectiva do Silogismo Aristotélico é que foram analisadas as abordagens sobre o Potencial Químico em dezessete fontes didáticas publicadas entre 1966 e 2016, a saber: Atkins e Paula (2008), Ball (2016), Callen (1985), Castellan (1995), Kondepudi (1998), Koretsky (2007), Levine (2002), Macedo e Luiz (1978), Moran (2014), Oliveira (2005), Pádua e Pádua (2006), Sears (1966), Sears e Salinger (1979), Smith (2007), Van Wylen (1995), Van Wylen e Sonntag (1975) e Zemansky (1978). Além desses livros, as definições também foram analisadas em dicionários, em uma Enciclopédia e em três sites da internet.

\section{RESULTADOS E DISCUSSÃO}

\subsection{Investigação das Definições de Potencial Químico em Livros Didáticos}

Neste item iremos analisar e comentar a apresentação e a abordagem sobre Potencial Químico em cada um dos livros pesquisados, segundo a ordem cronológica da publicação.

Sears (1966) definiu equilíbrio termodinâmico, porém não abordou equilíbrio químico nem Potencial Químico.

Van Wylen e Sonntag (1975) enfatizaram equilíbrio químico e a sua influência sobre a mudança da composição química de uma substância: "Quando o potencial químico de um componente é o mesmo em ambas as fases, não há tendência para uma transferência líquida de massa de uma fase para outra" (VAN WYLEN \& SONNTAG, 1975, p. 468).

Van Wylen e Sonntag (1975) afirmaram que Função de Gibbs Parcial Molar é sinônimo de Potencial Químico.

Macedo e Luiz (1978) apenas citaram que Potencial Químico é um coeficiente que aparece em equações de equilíbrio químico.

Zemansky (1978) fez o seguinte comentário sobre o Potencial Químico:

Consideremos agora o efeito exercido sobre a função de Gibbs quando se introduz na fase uma pequena quantidade de um dos componentes (por exemplo, o k-ésimo componente $A_{k}$ ), permanecendo constantes $T, P$ e os $n$ restantes. Se $d n_{k}$ moles de $A_{k}$ forem introduzidos, o efeito sobre a função de Gibbs estará expressado pela derivada parcial

$$
\mu_{k}=\frac{\partial G}{\partial n_{k}}
$$

onde $\mu_{k}$ é denominado potencial químico, do $k=e$ simo constituinte da fase em questão. O potencial químico de um componente é uma função de $T, P$ e de todos os $n$. Se uma substância não estiver presente em uma fase, isto não quer dizer que seu potencial químico seja nulo. O potencial químico é uma medida 
do efeito sobre a função de Gibbs quando uma substância é introduzida. Mesmo que a substância não esteja presente na fase, há sempre a possibilidade de introduzi-la, em cujo caso a função de Gibbs se modificaria e o valor de $\mu$ seria finito. (ZEMANSKY, 1978, p.523).

Zemansky (1978) também apresentou a relação entre Potencial Químico e Energia Molar de Gibbs. Entretanto, diferentemente de outros autores, Zemansky (1978) explora o Potencial Químico em diferentes fases de um sistema de componente único:

Neste caso simples, porém não trivial,

$$
G=\mu n
$$

e

$$
\mu=\frac{G}{n}=g
$$

isto é, o potencial químico é a função molar de Gibbs e depende unicamente de $T$ e $P$. (ZEMANSKY, 1978, p.524)

Desta forma, foi ressaltada a relação entre a energia molar de Gibbs e o Potencial Químico e a igualdade desses dois valores quando o sistema for formado por apenas um componente.

Zemansky (1978) ainda apresentou um exemplo em que a fase é constituída por uma mistura de gases perfeitos:

Neste caso temos, de acordo com a Seção 16.5,

$$
G=R T \sum n_{k}\left(\phi_{k}+\ln P+\ln x_{k}\right)
$$

que, quando comparada com a equação geral

$$
G=\sum n_{k} \mu_{k}
$$

Demonstra que o potencial químico de um gás perfeito em uma mistura de gases perfeitos é

$$
\mu_{k}=R T\left(\phi_{k}+\ln P+\ln x_{k}\right)
$$

que pode ser escrito em uma qualquer das duas formas:

$$
\stackrel{\mathrm{e}_{k}=R T\left(\phi_{k}+\ln P\right)}{\mu_{k}=g_{k}+R T \ln x_{k}}
$$

Donde pode concluir que nem sempre existe uma relação de igualdade entre a energia molar de Gibbs $(g)$ e o Potencial Químico $(\mu)$, uma vez que o valor do Potencial Químico é igual à energia molar de Gibbs acrescentada do termo $R T \ln x$.

Para o caso de fase formada por uma solução ideal, Zemansky (1978, p. 525) comenta que " $g_{k}$ é a função de Gibbs de $1 \mathrm{~mol}$ do $k$-ésimo constituinte no estado puro, expresso como 
uma função de $T$ e $P$ ”. Esta situação é análoga ao do caso anterior, com a diferença de que nesse caso é necessário fazer o cálculo para cada " $k$-ésimo" componente no estado puro.

Para o caso de fase constituída de uma solução diluída, temos que:

... a fração molar do solvente $x_{0}$ é muito maior do que cada uma das frações molares dos solutos $x_{1}, x_{2}, \ldots$, pode-se demonstrar que, para o solvente,

$$
\mu_{0}=g_{0}+R T \ln x_{0}
$$

onde $g_{0}$ é a função de Gibbs molar do solvente no estado puro, expresso como uma função de $T$ e $P$. Para qualquer um dos solutos,

$$
\mu_{k}=g_{0 k}+R T \ln x_{k}
$$

onde $g_{0 k}$ a uma função somente de $T$ e $P$, que depende, porém, da natureza do solvente, bem como da natureza do soluto. (ZEMANSKY, 1978, p. 525).

Finalmente, sobre sistemas compostos ainda encontramos o seguinte comentário: "Definindo certas funções, conhecidas como coeficiente de fugacidade e de atividade, é possível expressar os potenciais químicos dos componentes de uma mistura de gases reais, e também de soluções concentradas. Isto é além do escopo deste livro.” (ZEMANSKY, 1978, p. 525). Embora Zemansky (1978) não tenha definido Potencial Químico com profundidade, avaliamos que, do ponto de vista conceitual, consideramos satisfatórias suas abordagens sobre Potencial Químico.

Sears \& Salinger (1979) apresentaram inicialmente uma relação matemática para o Potencial Químico:

O potencial químico $\mu$ de cada gás na mistura é definido como

$$
\begin{gathered}
\mu \equiv R T(\ln P+\phi+\ln x) \\
g=R T(\ln P+\phi) \\
\mu=g+\ln x
\end{gathered}
$$

onde $g$ é a função de Gibbs específica à temperatura $T$ e à pressão total $P$.

(SEARS \& SALINGER, 1079, p.189).

Logo em seguida, Sears \& Salinger (1979) apresentaram a seguinte relação matemática para representar o potencial químico:

$$
\mu \equiv\left(\frac{\partial U}{\partial n}\right)_{S, V}
$$

Isto é, o potencial químico é a variação da energia interna por mol de substância acrescentada ao sistema em um processo a $S$ e $V$ constantes; a Eq. (8-8) pode ser escrita

$$
d U=T d S-p d V+\mu d n
$$

Esta equação é a forma geral da primeira e da segunda lei combinadas para um sistema $P V T$ aberto. De modo mais geral, se $X$ representa uma variável extensiva qualquer, correspondente ao volume $V$; e $Y$ representa a variável intensiva, correspondente à pressão $P$, o trabalho em um processo reversível diferencial é $Y d X$ e 


$$
d U=T d S-Y d X+\mu d n
$$

(8-12) (SEARS \& SALINGER, 1979, p. 191)

Sears \& Salinger (1979) fizeram uma abordagem puramente matemática do Potencial Químico e em função de outras grandezas diferentes da Energia Interna. Esse destaque quanto à abordagem matemática fica mais evidente com a citação que se segue:

"O potencial químico pode ser expresso em uma variedade de modos diferentes...

... Em resumo, temos as seguintes expressões para o potencial químico:

$$
\mu=-T\left(\frac{\partial S}{\partial n}\right)_{U, X}=\left(\frac{\partial F}{\partial n}\right)_{T, X}=\left(\frac{\partial G}{\partial n}\right)_{T, Y},
$$

(SEARS; SALINGER, 1979, p.192).

Callen (1985) apresentou o Potencial Químico por meio da diferencial da Energia Interna em relação ao número de moles:

$$
\left(\frac{\partial U}{\partial N_{j}}\right)_{S, V, N_{K}}=\mu_{j}
$$

Com relação aos vários potenciais eletroquímicos, não temos definições ou conceitos anteriores e somos livres para adotar a definição (equação 2.5) imediatamente. Por uma questão de brevidade, o potencial eletroquímico é frequentemente chamado simplesmente de potencial químico, e usaremos esses dois termos de forma intercambiável. (CALLEN, 1985, p. 35; 36, tradução nossa).

Em seguida, Callen (1985) associou o Potencial Químico à transferência de matéria:

Assim como a temperatura pode ser vista como uma espécie de "potencial" para fluxo de calor e a pressão pode ser vista como uma espécie de "potencial" para alterações de volume, o potencial químico pode ser visto como uma espécie de "potencial" para o fluxo de matéria. Uma diferença no potencial químico fornece uma "força generalizada" para o fluxo de matéria. (CALLEN, 1985, p.55, tradução nossa).

Observamos que, apesar de ser considerada válida a comparação entre 'temperatura' e 'fluxo de calor', assim como 'potencial químico' e 'fluxo de matéria', observamos que nestas duas análises, do ponto de vista da Lógica Aristotélica, foram usadas premissas distintas. Callen (1985) não apresentou uma definição, mas destacou a sua importância para a química teórica:

$$
d S=\frac{\mu_{i}^{2}-\mu_{i}^{1}}{T} d N_{i}^{1}
$$

Se $\mu_{i}^{1}$ for maior que $\mu_{i}^{2}, d N_{i}^{1}$ será negativo, pois o $d S$ deve ser positivo. Assim, a matéria tende a fluir de regiões de alto potencial químico para regiões de 
baixo potencial químico. Nos próximos capítulos, veremos que o potencial químico fornece a força generalizada não apenas para o fluxo de matéria de um ponto a outro, mas também para suas mudanças de fase e para reações químicas. O potencial químico, portanto, desempenha um papel dominante na química teórica. As unidades de potencial químico são joules por mole (ou qualquer unidade de energia desejada por mole). (CALLEN, 1985, p.55, tradução nossa)

Vale ressaltar que Correia et all (2008 e 2009) advertiram que o termo "fluxo" só deve ser utilizado quando a transferência de algo de um sistema a outro puder ser representado por uma grandeza vetorial.

Já Castellan (1995) abordou sobre Potencial Químico da seguinte forma:

É costume se usar o símbolo especial $\mu$ para a energia de Gibbs por mol; então, definimos

$$
\mu=\frac{G}{n}
$$

Portanto, para a energia de Gibbs molar do gás ideal, temos

$$
\mu=\mu^{0}(T)+R T \ln (p)
$$

Como .na Seç. 9.11, o símbolo $p$ na Eq. (10.47) representa um número puro que, quando multiplicado por $1 \mathrm{~atm}$, fornece o valor da pressão em atmosferas. O termo logarítmico na Eq. (10.47) é bastante grande e na maioria das circunstâncias não pode ser ignorado. (CASTELLAN, 1995, p. 227).

Dessa forma, Castellan (1995) também relacionou o Potencial Químico com 'energia de Gibbs molar' e, logo em seguida, relacionou Potencial Químico com equilíbrio químico de um sistema:

\begin{abstract}
A propriedade $\mu_{i}$ é chamada potencial químico da substância $i$. A matéria escoa espontaneamente de uma região de potencial químico alto para uma região de potencial químico mais baixo assim como a corrente elétrica escoa espontaneamente de uma região de potencial elétrico alto para uma de potencial elétrico mais baixo, ou como uma massa desloca-se espontaneamente de uma posição de potencial gravitacional alto para uma de potencial gravitacional mais baixo. Outro nome frequentemente dado para $\mu_{i}$ é tendência de escape da substância $i$. Se o potencial químico do componente de um sistema for alto, este componente terá maior tendência de escape, enquanto que, se o potencial químico for baixo, o componente terá uma pequena tendência de escape. (CASTELLAN, 1995, p.236).
\end{abstract}

Pode ser concluído que quanto menor a "tendência de escape" de um sistema, maior será a sua estabilidade.

Observa-se que Castellan (1995) não definiu Potencial Químico, mas o abordou por meio de relações matemáticas. 
Van Wylen (1995) também abordou o Potencial Químico por meio de uma representação matemática:

$$
d U=T d S-p d V
$$

Nesta equação observamos que a temperatura é a propriedade intensiva ou função potencial associada à entropia e a pressão é a propriedade intensiva associada ao volume. Suponhamos, agora, que ocorra uma reação química em que as quantidades dos componentes $A$ e $B$ mudem. Como poderíamos adequar esta relação de propriedades para essa situação? Podemos escrever, intuitivamente, a equação

$$
d U=T d S-p d V+\mu_{A} d n_{A}+\mu_{B} d n_{B}
$$

onde $\mu_{A}$ é a propriedade intensiva ou função potencial associada a $n_{A}$. Analogamente, $\mu_{B}$ é a função potencial associada a $n_{B}$. Esta função potencial é denominada potencial químico. (VAN WYLEN, 1995, p. 375).

e continua com sua análise puramente matemática, ao observar que:

... como as propriedades molares parciais são definidas à temperatura e pressão constantes, as quantidades $\mu_{A}=\left(\frac{\partial G}{\partial n_{A}}\right)_{T, p, n_{B}}$ e $\mu_{B}=\left(\frac{\partial G}{\partial n_{B}}\right)_{T, p, n_{A}}$ são funções de Gibbs molares dos dois componentes. Isto é, o potencial químico é igual à função de Gibbs molar parcial. (VAN WYLEN, 1995, p. 276).

Vale ressaltar que o uso da terminologia "função de Gibbs molar parcial" ao invés da "função molar de Gibbs" foi devido à referência em que o sistema é constituído por mais de uma componente. Dessa forma, a equivalência é sempre garantida, independentemente do número de partículas, pois, no caso de um sistema simples, a função de Gibbs molar parcial seria equivalente a função molar de Gibbs. Observa-se também que Van Wylen (1995) igualou potencial químico à função de Gibbs molar parcial, em vez de igualar Potencial Químico à variação da Energia Livre de Gibbs para determinado componente, em relação ao número de moles, à Pressão e Temperatura constantes, mantendo constante também o número de moles dos outros componentes do sistema. Van Wylen (1995) fez uma abordagem matematizada sobre Potencial Químico.

Entendemos que a relação que Van Wylen (1995) estabeleceu entre Potencial Químico e Temperatura, além de evidenciar que Temperatura pode ser vista sob outra perspectiva, contribuindo para facilitar a compreensão e a significação de Potencial Químico.

Kondepudi (1998), diferente dos autores mencionados até então, fez uma abordagem histórica quanto ao surgimento do Potencial Químico: 
Josiah Willard Gibbs introduziu a ideia de potencial químico em sua famosa obra On the Equilibrium of Heterogeneous Substances, publicada em 1875 e 1878 [4-6]. Gibbs publicou seu trabalho na Transactions of the Connecticut Academy of Sciences, uma revista que não foi amplamente lida. Esse trabalho de Gibbs permaneceu em relativa obscuridade até ser traduzido para o alemão por Wilhelm Ostwald (1853-1932) em 1892 e para o francês por Henry-Louis Le Chatelier (1850-1936) em 1899 [I]. Grande parte da termodinâmica de equilíbrio atual pode ser rastreada até esse importante trabalho de Gibbs. Gibbs considerou um sistema heterogêneo (Fig. 4.1) que consistia em várias partes homogêneas, cada uma contendo várias substâncias $S_{1}, S_{2}, \ldots, S_{n}$, das massas $m_{1}, m_{2}, \ldots, m_{n}$. Sua consideração inicial não incluiu reações químicas entre essas substâncias, mas restringiu-se à troca entre as diferentes partes homogêneas do sistema. Argumentando que a mudança na energia $d U$ de uma parte homogênea deve ser proporcional às mudanças nas massas das substâncias $d m_{1}, d m_{2}, \ldots, d m_{n}$, Gibbs introduziu a equação

$$
d U=T d S-p d V+\mu_{1} d m_{1}+\mu_{2} d m_{2}+\cdots+\mu_{m} d m_{m}
$$

(KONDEPUDI, 1998, p. 104; 105, tradução nossa).

Essa citação é interessante porque contextualiza o surgimento do Potencial Químico, além de destacar que a ciência é construída em etapas e que uma grandeza tal qual conhecemos na atualidade sofreu alterações teóricas para que se adequar melhor à realidade. Por exemplo, hoje não se utiliza mais o número de massa e sim o número de moles.

Kundepudi não definiu Potencial Químico, mas apresentou uma situação de desequilíbrio termodinâmico numa situação em que um sistema for constituído por substâncias de potenciais químicos distintos:

\begin{abstract}
A ideia de potencial químico acaba tendo ampla aplicabilidade. Vários outros processos irreversíveis que podem ser descritos usando um potencial químico serão discutidos no Capítulo 10. Quando os potenciais químicos de partes adjacentes de um sistema são desiguais, a difusão da matéria ocorre até que os potenciais químicos das duas partes se tornem iguais. O processo é semelhante ao fluxo de calor que ocorre devido a uma diferença de temperatura. A difusão é outro processo irreversível para o qual podemos obter a taxa de aumento da entropia em termos dos potenciais químicos. (KONDEPUDI, 1998, p. 114, tradução nossa).
\end{abstract}

Observa-se que o uso do termo "fluxo de calor", conforme argumenta Correia et all (2008 e 2009), constitui num erro lógico quando o seu uso estiver associado a uma grandeza escalar.

A melhor representação matemática que contribui para o entendimento do significado de Potencial Químico foi encontrada em Levine (2002, p. 210). Esse livro descreve o Potencial Químico como uma propriedade intensiva e representado pela seguinte equação $\mu_{P_{2}}=\mu_{P_{1}}+$ $R T \ln \left(\frac{P_{2}}{P_{1}}\right)$, para um Sistema que evoluiu isotermicamente do estado de $P_{1}$ a $P_{2}$. Para deduzir a 
equação acima, Levine (2002) apresentou a equação diferencial da Energia Livre de Gibbs $d G=-S d T+V d P$ e dividiu pelo número de moles $(n)$ e, considerou também que $\mu=\frac{G}{n}=$ $G_{m}$ para um gás puro e ideal. E ainda argumentou:

Para um gás puro, $\mu=G_{m}=H_{m}-T S_{m}$, sendo $H_{m}$ independente da Pressão e que a dependência de $\mu$ com a Pressão é devido à dependência de $S_{m}$ com a Pressão. No limite da Pressão nula, e, portanto, com o Volume tendendo a infinito, a Entropia de um gás ideal tende a infinito, levando $\mu$ tender ao infinito. (LEVINE, 2002, p. 210).

Levine (2002) também fez uma discussão bem detalhada da variação do Potencial Químico.

Oliveira (2005) abordou sobre o Potencial Químico da seguinte forma:

Sendo $U$ também uma função de $N$, é conveniente definir uma grandeza que esteja associada à variação da energia quando se faz um incremento do número de moles da substância pura que compõe o fluido. Essa grandeza é denominada potencial químico $\mu$ e é definida por

$$
\mu=\frac{\partial U}{\partial N}
$$

de modo que podemos escrever a conservação da energia em forma diferencial como

$$
d U=T d S-p d V+\mu d N
$$

Oliveira (2005) apenas apresentou uma relação matemática para Potencial Químico, sem defini-lo.

Pádua e Pádua (2006) abordaram sobre Potencial Químico apenas como um parâmetro em relações matemáticas da Termodinâmica. Ou seja, não o definiu.

Smith (2007), embora não tenha definido Potencial Químico, foram apresentadas algumas igualdades de relações que representem equilíbrio químico:

Assim,

$$
\mu_{i}^{\alpha}=\mu_{i}^{\beta}, \quad i=1,2, \ldots, N
$$

onde $N$ é o número de espécies presentes no sistema. A utilização sucessiva desse resultado em pares de fases permite a sua generalização para múltiplas fases; para $\pi$ fases:

$$
\mu_{i}^{\alpha}=\mu_{i}^{\beta}=\cdots=\mu_{i}^{\pi}, i=1,2, \ldots, N
$$

Uma dedução similar, porém, mais abrangente, mostra (como foi admitido) que, para o equilíbrio, $T$ e $P$ têm que ser as mesmas em todas as fases.

Assim, múltiplas fases nas mesmas $T$ e $P$ estão em equilíbrio de forma que o potencial químico de cada espécie é o mesmo em todas as fases. (SMITH, 2007, p. 204). 


\section{A DEFINIÇÃO DE POTENCIAL QUÍMICO EM FONTES DIDÁTICAS}

Koretsky (2007) fez a seguinte abordagem:

O potencial químico é um conceito abstrato; ele não pode ser medido diretamente. Porém, a relação entre o potencial químico e o transporte de massa é idêntica à relação entre a temperatura e o transporte de energia, ou a pressão e o transporte de momento. Este conceito é ilustrado na Figura 6.14. Na Figura 6.14a, vemos dois sistemas a temperaturas diferentes. Se eles forem colocados em contato, haverá transferência de energia, em forma de calor, da temperatura alta para a temperatura baixa até que as temperaturas fiquem iguais e o equilíbrio térmico seja estabelecido. Poderíamos tranquilamente chamar a temperatura de "potencial térmico", uma vez que ela fornece a força motriz para o equilíbrio térmico. Porém, já conhecemos essa propriedade, a partir da nossa experiência física, como temperatura e, por esta razão, mantemos este nome. A Figura 6.14b, mostra a relação análoga entre o potencial químico e a difusão. Aqui vemos dois sistemas com potenciais químicos diferentes para a espécie química $i$. Neste caso, haverá transporte da espécie química $i$ do potencial químico maior para o potencial químico menor até que os potenciais químicos se igualem e o equilíbrio químico seja alcançado. Se conhecermos o $\mu_{i}$ de cada fase, saberemos de que modo a espécie $i$ tenderá a se transferir. A maior dificuldade para se entender $o$ potencial químico é que ele é um conceito abstrato, enquanto que a $T$ e a $P$ são propriedades mensuráveis, com as quais temos experiência direta. Entretanto, podemos aplicar o conceito de potencial químico para aprender sobre a força motriz para a transferência de espécies químicas, da mesma maneira que associamos a temperatura ao transporte de energia. (KORETSKY, 2007, p. 262).

Esta analogia facilita a compreensão das grandezas envolventes. Entretanto as premissas que relacionam 'potencial químico' e o 'transporte de massa' é distinta da premissa que relaciona 'temperatura' e o 'transporte de energia' e também distinta da premissa que relaciona a 'pressão' e o ‘transporte de momento'.

Mais à adiante, Koretsky (2007) apresentou a relação do Potencial Químico com o equilíbrio químico:

Acabamos de estudar que o potencial químico fornece o critério para o equilíbrio químico da substância $i$ entre as fases $\alpha$ e $\beta$ em um sistema multicomponente:

$$
\mu_{i}^{\alpha}=\mu_{i}^{\beta}
$$

Ele é uma propriedade termodinâmica auxiliar, diferente das propriedades termodinâmicas mensuráveis temperatura e pressão, que fornecem os critérios para o equilíbrio térmico e o equilíbrio mecânico, respectivamente. Embora seja um conceito abstrato, o potencial químico é útil, uma vez que fornece um critério simples pra o equilíbrio químico de uma dada substância $i$.

Infelizmente, em termos práticos, observa-se que o potencial químico apresenta um comportamento matemático inconveniente. (KORETSKY, 2007, p. 273). 
Entendemos que a citação acima faz uso de mesma premissa para estabelecer uma analogia entre as energias térmica, mecânica e química.

Assim como as outras fontes analisadas neste trabalho, Koretsky (2007) não apresentou uma definição formal para o potencial Químico, mas salientou que sua aplicação pode apresentar algumas dificuldades.

Atkins \& Paula (2008) abordaram sobre o Potencial Químico relacionando-o com a Energia Molar de Gibbs:

Toda a nossa análise será baseada na energia de Gibbs de uma substância, em particular na sua energia de Gibbs molar, $G_{m}$. Na verdade, essa grandeza termodinâmica é tão importante neste capitulo, bem como no restante do livro, que daremos a ela um nome e um símbolo especiais: potencial químico $\mu(\mathrm{mi})$. Para sistemas com um componente apenas, a 'energia de Gibbs molar' e o 'potencial químico' são equivalentes, ou seja, $\mu=G_{m}$, mas no Cap. 5 veremos que o potencial químico tem um significado mais abrangente e uma definição mais geral. (ATKINS; PAULA, 2008, p. 111).

Pode ser observado nesta fonte pesquisada que Potencial Químico e Energia Molar de Gibbs são grandezas distintas para sistemas compostos. Um dos motivos para o Potencial Químico ser uma grandeza muito importante é a sua relação com o equilíbrio químico de um sistema. Isso fica evidente em um trecho apresentado por Atkins \& Paula (2008), ao afirmarem que: “o nome 'potencial químico' também tem um significado próprio: à medida que desenvolvermos o seu estudo, veremos que $\mu$ corresponde a uma medida do potencial (da tendência) que uma substância apresenta de sofrer uma mudança em um dado sistema" (Atkins \& Paula, 2008, p. 111). Dessa forma, a clareza da definição do Potencial Químico é primordial, principalmente quando estiver sendo analisado o equilíbrio de reações químicas.

Atkins \& Paula (2008) ainda afirmaram:

A fundamentação da nossa discussão é o seguinte resultado da Segunda Lei da termodinâmica: no equilíbrio, o potencial químico de uma substância é o mesmo em toda a amostra, qualquer que seja o número de fases presentes. Quando as fases líquida e sólida de uma substância estão em equilíbrio, o potencial químico da substância é o mesmo em qualquer ponto do sistema. Para tornar evidente a validade dessa observação, imaginemos um sistema em que o potencial químico de uma substância seja $\mu_{1}$, num certo ponto e $\mu_{2}$ em outro ponto. Os pontos podem estar no interior de uma fase ou em fases diferentes. Quando uma quantidade $d n$ da substância é transferida do primeiro para o segundo ponto, a energia de Gibbs do sistema varia de $-\mu_{1} d n$, quando a substância é removida do ponto 1 , e varia de $+\mu_{2} d n$ quando a substância é adicionada no ponto 2. A variação total é então $d G=\left(\mu_{2}-\mu_{1}\right) d n$. Se o potencial químico no ponto 1 for mais elevado do que no ponto 2 , a transferência da substância é acompanhada por uma diminuição de $G$ e, por 
isso, tem tendência a ocorrer espontaneamente. Somente se $\mu_{1}=\mu_{2}$ não haverá mudança de $G$, e somente então o sistema estará em equilíbrio. A partir desse resultado concluímos que a temperatura de transição, $T_{\text {trs }}$, é a temperatura em que os potenciais químicos das duas fases são iguais. (ATKINS; PAULA, 2008, p. 111).

Essa afirmação é extremamente importante, porque ao invés de calcular o Potencial Químico em cada componente do sistema, no caso de um sistema em equilíbrio, calcula-se o valor de apenas uma componente e esse valor representará toda amostra, independentemente do número de fases apresentadas. A importância desta afirmação de Atkins \& Paula (2008) está na sua contribuição para facilitar a compreensão do significado de Potencial Químico.

Todavia, vimos apresentar duas observações: foi afirmado que se basearam na Segunda Lei da Termodinâmica, mas não explicaram o porquê dessa conclusão; e, ao apresentarem um exemplo, estes autores não relacionaram com o cotidiano, apesar de entendermos que uma exemplificação matemática pode facilitar a sua compreensão.

Atkins \& Paula (2008) também analisaram a influência da Temperatura de uma substância sobre o Potencial Químico:

Nas temperaturas baixas e desde que a pressão não seja muito baixa, a fase sólida de uma substância tem um potencial químico menor que o das outras fases e é, portanto, a fase mais estável. Entretanto, como os potenciais químicos das fases se alteram com a temperatura, e se alteram de maneiras diferentes, é possível que, ao se elevar a temperatura, o potencial químico de outra fase (sólida, líquida ou vapor) fique mais baixo do que o potencial da fase sólida. Quando isso ocorre, há uma transição para a segunda fase, desde que não haja impedimento cinético.

A dependência da energia de Gibbs com a temperatura se exprime em função da entropia do sistema pela eq. $3.50\left[\left(\frac{\partial G}{\partial T}\right)_{P}=-S\right]$. Como o potencial químico de uma substância pura é a energia de Gibbs molar da substância, vem que

$$
\left(\frac{\partial \mu}{\partial T}\right)_{P}=-S_{m}
$$

Essa relação mostra que quando a temperatura se eleva, o potencial químico de uma substância pura diminui: a entropia molar é sempre positiva $\left(S_{m}>0\right)$ para todas as substâncias, de modo que o coeficiente angular da curva de $\mu$ contra $T$ é sempre negativo. (ATKINS; PAULA, 2008, p. 111).

Obviamente, isso ocorre quando a substância não se encontra em equilíbrio.

Finalmente, Atkins \& Paula (2008) apresentaram um caso especial da equação de Gibbs-

Duhem e a sua relação com o Potencial Químico:

Esta equação é um caso especial da equação de Gibbs-Duhem:

$$
\sum_{i} n_{i} \mu_{i}=0
$$


O significado da equação de Gibbs-Duhem é que o potencial químico de um componente numa mistura não pode se alterar independentemente dos potenciais químicos dos outros componentes. Numa mistura binária, se um dos potenciais químicos aumenta, o outro deve diminuir, com as duas variações relacionadas por

$$
d \mu_{B}=-\frac{n_{A}}{n_{B}} d \mu_{A}
$$

Atkins \& Paula (2008) não definiram Potencial Químico, mas a abordagem matemática foi detalhada e satisfatória.

Moran (2014) apresentou o Potencial Químico por meio da função de Gibbs molar parcial:

Das propriedades molares parciais, a função de Gibbs molar parcial é particularmente útil na descrição do comportamento de misturas e soluções. Essa quantidade desempenha um papel central nos critérios de equilíbrio químico e de fase (Cap. 14). Devido à sua importância no estudo de sistemas multicomponentes, a função parcial de Gibbs molar do componente $i$ recebe um nome e símbolo especiais. É chamado de potencial químico do componente $i$ e simbolizado por $\mu_{i}$ :

$$
\mu_{i}=\bar{G}_{i}=\left(\frac{\partial y}{\partial n_{i}}\right)_{T, P, \mu_{j}}
$$

Como temperatura e pressão, o potencial químico $\mu_{i}$ é uma propriedade intensiva.

Aplicando a Eq. 11.103 junto com a Eq. 11.107, a seguinte expressão pode ser escrita:

$$
G=\sum_{i=1}^{f} n_{i} \mu_{i}
$$

(11.108) (MORAN, 2014, p. 706, tradução nossa).

Dessa forma, é apresentada uma equação para o Potencial Químico e um campo de estudo em que ele é útil. Logo em seguida, Moran (2014) apresenta uma equação mais geral para o Potencial Químico:

$$
\mu^{*}=R T \ln (p)+C(T)
$$

Onde $C(T)$ é uma função da integração. Como a pressão $p$ pode assumir valores de zero a mais infinito, o termo $\ln (p)$ dessa expressão e, portanto, o potencial químico, tem uma faixa inconveniente de valores de menos infinito a mais infinito. A equação 11.120 também mostra que o potencial químico pode ser determinado somente dentro de uma constante arbitrária. (MORAN, 2014, p. 709, tradução nossa). 
É importante ressaltar que Moran (2014) não define Potencial Químico, mas relata que o termo logaritmo da pressão pode atrapalhar a sua aplicação, uma vez que esse fator assume valores extremos muito grandes.

Ball (2016) definiu o Potencial Químico de uma substância em função da Energia Livre de Gibbs:

Até agora focalizamos as mudanças nos sistemas que são medidas com base em variáveis físicas como pressão, temperatura e volume, dentre outras. Mas em reações químicas as substâncias mudam sua forma química. Precisamos começar a focalizar a identidade química dos materiais e o modo pelo qual eles podem se transformar no decorrer de um processo.

Assumimos que o número de mols, $n$, de uma substância permanece constante em todas as transformações pelas quais ela passou até agora. Todas as derivadas parciais devem ter um subscrito $n$ no lado direito para indicar que a quantidade de material permanece constante: por exemplo, $\left(\frac{\partial U}{\partial T}\right)_{T, n}$. Entretanto, não há razão para não considerarmos uma derivada em relação à quantidade $n$.

Devido à importância da energia livre de Gibbs quando consideramos a questão da espontaneidade, a maioria das derivadas em relação a $n$ diz respeito a $G$. O potencial químico de uma substância, $\mu$, é definido como a variação na energia livre de Gibbs em relação à quantidade, a pressão e temperatura constantes:

$$
\left(\frac{\partial G}{\partial n}\right)_{T, P} \quad \text { (BALL, 2016, p. 108). }
$$

É notório que a expressão acima não apresenta significação para $\mu$. Todavia, na página 110 desse mesmo livro, Ball (2016) apresentou a seguinte definição:

Potencial químico é uma medida do quanto uma espécie "deseja" sofrer uma mudança física ou química. Se duas ou mais substâncias estão presentes em um sistema e têm diferentes potenciais químicos, algum processo deve ocorrer para igualar esses potenciais. Desse modo, potencial químico nos leva a pensar em reações químicas e em equilíbrio químico. (BALL, 2016, p. 110).

Entendemos que esta foi uma das melhores abordagens que encontramos no decorrer desta nossa pesquisa, mesmo não que não seja uma definição propriamente dita. Observou-se que Ball (2016) apresentou uma equação para o Potencial Químico de sistemas que têm mais de um componente e exibe uma equação denominada como "equação fundamental da termodinâmica química":

Para sistemas com mais de um componente químico, temos de rotular o potencial químico (um número ou uma fórmula química como subscrito) para especificar a qual componente se refere. O potencial químico para um componente $\mu_{i}$ indica que apenas a quantidade $n_{i}$ do iésimo componente varia, 
e as quantidades de todos os outros componentes $n_{j}, j \neq i$, permanecem constantes. Assim, a Equação 4.46 pode ser escrita como

$$
\mu_{j}=\left(\frac{\partial G}{\partial n_{i}}\right)_{T, p, n_{j}}(j \neq i)
$$

Agora, se quisermos considerar a variação infinitesimal de $G$, precisamos também considerar possíveis variações nas quantidades de substância. A expressão geral $d G$, agora, se torna

$$
\begin{gathered}
d G=\left(\frac{\partial G}{\partial n_{i}}\right)_{p, n, S} d T+\left(\frac{\partial G}{\partial n_{i}}\right)_{T, n, S} d P+\sum_{i}^{j \neq i}\left(\frac{\partial G}{\partial n_{i}}\right)_{T, p, n_{j}} d n_{i} \\
\mathrm{Ou} \quad d G=-S d T+V d P+\sum_{i}^{j \neq i} \mu_{i} d n_{i}
\end{gathered}
$$

Onde a somatória tem tantos termos quantas forem as diferentes substâncias no sistema. Muitas vezes, nos referimos à Equação 4.48 como a equação fundamental da termodinâmica química, uma vez que ela engloba, além de todas as variáveis de estado do sistema, as quantidades de substância.

O potencial químico $\mu_{i}$ é o primeiro exemplo de uma quantidade molar parcial. Ele expressa a variação de uma variável de estado, a energia livre de Gibbs, em relação à quantidade molar. Para substâncias puras, o potencial químico é simplesmente igual à variação de energia livre de Gibbs do sistema, à medida que a quantidade de material varia. Para sistemas com mais de um componente, o potencial químico não é igual à variação da energia livre do material puro, porque cada componente interage com os outros, o que afeta a energia total do sistema. Se todos os componentes fossem ideais, isto não aconteceria, e as quantidades molares parciais seriam as mesmas para qualquer componente do sistema. (BALL, 2016, p. 109).

Foi observado que Ball (2016) fez uma discussão bastante interessante a respeito do Potencial Químico.

Pelos materiais didáticos analisados nesta pesquisa, podemos concluir que a maioria dos livros pesquisados não se preocupou com a definição do Potencial Químico e mesmo quando tencionaram apresentar uma definição, geralmente citaram relações matemáticas e algumas formas de aplicação.

Em suma, observamos que as definições apresentadas sobre Potencial Químico em livros didáticos foram superficiais, que as discussões sobre a variação da sua intensidade foram satisfatórias, que a discussão da sua conservação foi pouco abordada, porém relevante, e que a discussão dos seus valores máximo e mínimo, embora insipiente, também foi satisfatória.

\subsection{Definições em enciclopédias}

Embora as enciclopédias estejam em desuso, essa fonte de pesquisa era bastante utilizada como referência para se apropriar do significado de determinado verbete. 


\section{A DEFINIÇÃO DE POTENCIAL QUÍMICO EM FONTES DIDÁTICAS}

A Enciclopédia Ciência Ilustrada (1972) se destaca pela sua abordagem científica, a exemplo da afirmativa: "uma das primeiras aproximações ao conceito de Potencial Químico é o calor de reação, que é a quantidade de energia térmica que será emitida ou absorvida durante determinada reação química" (Ciência Ilustrada, p. 4376). Mais adiante, nessa mesma página, foi encontrada a seguinte afirmação: "pode-se então dizer que o calor desenvolvido em qualquer reação constitui uma indicação do seu potencial químico". Mas fez a seguinte ressalva:

O calor de reação, contudo, é só uma aproximação do conceito de potencial químico, e só indica o sentido em que se processa espontaneamente uma reação química em casos muito particulares.

Na página 4377, encontra-se a seguinte observação:

O potencial químico é, portanto, uma função termodinâmica que permite prever qual será o comportamento químico de certo sistema.

Embora seja uma função bastante complexa, porque depende de muitas variáveis físicas e químicas, ela pode ser simplificada, ao se considerarem reações sob pressão muito baixa ou entre substâncias que se encontram na mesma fase; ou, ainda, quando se consideram transformações entre fases sucessivas de equilíbrio.

A partir desta citação, a enciclopédia não teceu mais comentários sobre o Potencial Químico nesta mesma caixa de texto, mas faz uma discussão muito interessante e resumida acerca do Calor, do Trabalho, da Energia Interna, da Entalpia, da Entropia, da Energia de Helmholtz e da Energia Livre de Gibbs. Essa abordagem sugere que o Potencial Químico depende dessas energias. Entretanto, o destaque foi mais relevante para as energias potenciais. Essa dependência fica evidente no comentário dentro de uma caixa de textona página 4378 dessa mesma enciclopédia:

Em resumo, pode-se dizer que a variação da entalpia só pode representar o potencial químico quando a entropia se mantém constante. E que a variação de entropia indica o sentido de uma reação somente quando a energia interna do sistema considerado não varia.

Apesar da tentativa de apresentar de uma significação para o Potencial Químico, houve uma abordagem muito abrangente. O que é óbvio, devido à característica do Conhecimento Enciclopédico. Mas, entendemos que por essa explanação um estudante ainda teria dificuldades para inferir sobre o significado acerca do Potencial Químico. 


\subsection{Definições em Sites da Internet}

É de trivial constatação que a utilização de computadores, celulares, entre outros meios de acesso à internet está cada vez mais difundida entre a população em geral. Dessa forma, o método de pesquisas mudou bastante nestes últimos anos. Nesse sentido, fizemos uma busca pela definição do Potencial Químico em alguns sites populares para analisar a qualidade das informações que os estudantes vêm tendo acesso.

Encontramos no site Wikipedia ${ }^{i}$ a seguinte definição para o Potencial Químico:

Em termodinâmica, potencial químico $\mu$ de um elemento, também conhecido como a energia livre parcial molar, é uma forma de energia potencial que pode ser absorvida ou liberada durante uma Reação química. Também pode ser importante durante uma Transição de fase. O potencial químico de uma espécie numa mistura pode ser definido como a inclinação da energia livre do sistema no que diz respeito a uma alteração no número de moles de apenas aquela espécie. Assim, é uma derivada parcial da energia livre no que diz respeito à quantidade da espécie, todas as outras concentrações de espécies presentes na mistura mantendo-se constante, e à uma temperatura constante. Quando a pressão é constante, o potencial químico é a Energia livre de Gibbs molar. Em condições de Equilíbrio químico ou em fase de equilíbrio, a soma total dos potenciais químicos é zero, assim como a energia livre está no mínimo.

Vale salientar que Potencial Químico não pode ser considerado como "uma forma de energia potencial", porque Potencial Químico não é uma energia como a Energia Interna, a Entalpia, o Potencial de Helmholtz nem como o Potencial de Gibbs.

No site WikiCiências ${ }^{i i}$ encontramos uma discussão puramente matemática:

Se o sistema tiver um único componente (gás, líquido ou sólido puros) o potencial químico, $\mu$, é definido como a energia livre molar, a determinada pressão e temperatura:

$$
\mu=\frac{G}{n}
$$

em que a energia livre, $G$, é uma função da pressão, $p$, da temperatura absoluta, $T$, e do número de moles $n$, ou seja, $G=G(p, T, n)$.

Para sistemas com vários componentes, $G=G\left(p, T, n_{l}, n_{2}, \ldots n_{i}, \ldots\right)$, e o potencial químico de cada componente define-se como a energia livre molar parcial:

$$
\mu=\left(\frac{\partial G}{\partial n_{i}}\right)_{T, p, n_{j} \neq, n_{i}}
$$

que é a taxa de variação da energia livre causada por uma variação do número de moles do componente $i$, mantendo constantes a pressão, temperatura e o número de moles das outras espécies, isto é, a derivada parcial de $G$ em ordem a $n_{i}$.

Das propriedades físicas de $G$, pode estabelecer-se a expressão:

$$
G\left(p, T, n_{1}, n_{2}, \ldots, n_{i, \ldots}\right)=n_{1} \mu_{1}+n_{2} \mu_{2} \ldots+n_{i} \mu_{i} \ldots
$$

que se reduz à definição (2) para um único componente. 
Dessa forma, assim como ocorreu na maioria das outras fontes pesquisadas, não foi encontrada uma definição satisfatória no site acima.

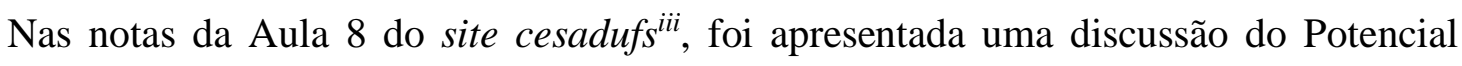
Químico similar à que foi apresentada em outros sites. Mas, esse site, apresenta a condição em que o Potencial Químico não pode ser igual à variação da energia livre do material puro, quando se tratar de um sistema com mais de uma componente:

Para sistemas com mais de um componente, o potencial químico não é igual a variação de energia livre do material puro, porque cada componente interage com os outros, o que afeta a energia total do sistema. Se todos os componentes fossem ideais, isto não aconteceria, e as quantidades molares parciais seriam as mesmas para qualquer componente do sistema.

Finalmente, este site apresentou uma discussão que tende a facilitar a compreensão do significado de Potencial Químico:

\begin{abstract}
Em síntese, podemos dizer que o potencial químico é uma medida do quanto uma espécie "deseja" sofrer uma mudança física ou química. Se duas ou mais substâncias estão presentes em um sistema e têm diferentes potenciais químicos, algum processo deve ocorrer para igualar esses potenciais. Desse modo, potencial químico nos leva a pensar em reações químicas em equilíbrio químico.
\end{abstract}

Apesar de esta explanação estar apresentada com superficialidade, entendemos que esta abordagem está entre as melhores dentre os sites pesquisados.

De modo geral, as definições que encontramos na internet, embora bastante resumidas, estão similares, quanto ao nível, às definições apresentadas nos livros pesquisados.

No século passado, o dicionário era bastante consultado para se adquirir uma noção acerca de determinado tema. No dicionário Aurélio, por exemplo, foi encontrada a definição para Potencial Químico, como sendo "a função de Gibbs parcial molar de um componente num sistema" (AURÉLIO, 1986, p. 1374). Pelas discussões acima, esta definição pode estar incompleta. 


\section{A DEFINIÇÃO DE POTENCIAL QUÍMICO EM FONTES DIDÁTICAS}

\section{CONCLUSÃO}

Mesmo que esta pesquisa tenha sido feita em fontes publicadas num intervalo de tempo de 50 anos, nenhum dos livros nem nenhum dos sítios da internet apresentou uma definição precisa, clara e completa de Potencial Químico. Da análise a partir do Silogismo Aristotélico, percebemos que a maioria das fontes pesquisadas não considerou as mesmas premissas lógicas nem utilizou elementos fundamentais para apresentar uma definição consistente. Ou seja, observou-se, nos livros consultados que foi dada importância para o Potencial Químico de determinado sistema para o controle de reações químicas e transporte de matéria, bem como para a sua relação com o equilíbrio termodinâmico, priorizando uma abordagem puramente matemática. Acreditamos que não houve objetivo em definir, nem mesmo de exemplificar, mas em descrever matematicamente uma função que o representasse. Entendemos que a apresentação da relação matemática de uma grandeza seja importante, mas, não suficiente para a sua compreensão.

Conclui-se que a definição de Potencial Químico nas fontes pesquisadas deixou a desejar e que poucos autores aprofundaram a discussão sobre a sua conservação. Notou-se escassez de abordagens sobre os valores máximo e mínimo do Potencial Químico e notou-se também que as discussões sobre a variação da sua intensidade foram superficiais.

Considerando que a definição de Potencial Químico foi imprecisa nestas fontes pesquisadas, apresentaremos aqui uma proposta de definição.

Para substâncias puras, com apenas um componente, o Potencial Químico é numericamente equivalente à Energia Livre de Gibbs por mol, ou seja, é equivalente à Função Molar de Gibbs. Todavia, essa equivalência cai por terra, caso exista mais de um componente no sistema, pois cada componente interagiria de alguma forma com os outros componentes, o que poderia afetar a energia total do sistema, levando a uma identidade diferente da imaginada para um sistema ideal. Dessa forma, no caso geral, ao invés da equivalência com a Energia Molar de Gibbs, haveria uma equivalência com a Função Molar Parcial de Gibbs, em que os valores do Potencial Químico variem de menos infinito até mais infinito. Por outro lado, a variação do valor do Potencial Químico para transformações isotérmicas de gases reais e a uma pressão inicial de 1 bar é

$$
\Delta \mu=R T \ln P
$$




\section{A DEFINIÇÃO DE POTENCIAL QUÍMICO EM FONTES DIDÁTICAS}

Em suma, Potencial Químico de um sistema representa a sua habilidade para a ocorrência de transformações químicas em seus componentes. Quando existem substâncias com Potenciais Químicos distintos em um único sistema, esse sistema fica sujeito a alguma transformação para que as substâncias de maior Potencial Químico percam energia química para as substâncias de menor Potencial Químico, até atingir o equilíbrio químico. Finalmente, a intensidade do Potencial Químico admite valores desde menos infinito até mais infinito, o que dificulta a análise da validade e da exclusão de determinados valores.

Após a análise dessas fontes didáticas pode-se inferir que apesar da definição de Potencial Químico ter ficado a desejar, foram encontradas discussões interessantes que contribuem a um bom entendimento do seu significado.

Embora tenhamos observado uma abordagem superficial das características do Potencial Químico de um Sistema, em detrimento do aprofundamento das definições, entendemos que são compreensíveis tais dificuldades e limitações quanto às definições de quaisquer grandezas com características potenciais, o que exige a definição de um valor de referência. Todavia, acreditamos que para uma real compreensão das grandezas da Física, uma compreensão teórica torna indispensável.

Considerando que as relações matemáticas do Potencial Químico nestas fontes pesquisadas envolvem variações de energias potenciais de sistemas fechados, este grupo de pesquisa visa apresentar, como perspectiva futura, as suas análises de fontes didáticas que abordam a definição de Potencial Químico associada a energia potencial para sistemas abertos.

\section{REFERÊNCIAS}

ARISTÓTELES. Segundos Analíticos: Livro I. Tradução, introdução e notas de Lucas Angioni. Campinas: IFCH/UNICAMP - Setor de Publicações, fevereiro de 2004. (Clássicos da Filosofia: Caderno de Tradução no 7 ).

ATKINS, P.; PAULA, J. Físico-Química. 2a . ed. 24a impressão. Rio de Janeiro: LTC, 2008.

AURÉLIO et al. Novo Dicionário Aurélio da Língua Portuguesa. 8. ed. Rio de Janeiro: Editora Nova Fronteira, 1986.

BALL, D. W. Físico-Química. São Paulo: Cengage Learning, 2016.

BRASIL. (s.d.). Brasil Escola. Disponível em Conteúdos de Química para o ENEM:< https://brasilescola.uol.com.br/quimica/conteudos-quimica-para-enem.htm.>. Acesso em 02 jan 2021, 
CALLEN, H. B. Thermodynamics and an Introduction to Thermostatistics, $2^{\mathrm{a}}$ Edition, Republic of Singapore: Editora John Wiley \& Sons, 1985.

CASTELlan, G. Fundamentos de Físico-Química. Rio de Janeiro: LTC, 1995.

Ciência Ilustrada, v. 10. São Paulo: Editora Abril Cultural, p. 4376-4379, 1972.

CORREIA, J.J.; MAGALHÃES, L. D. R.; LIMA, L. S. Obstáculos Epistemológicos e o Conceito de Calor. Sitientibus Série Ciências Físicas. 2008. v. 4, p. 1-10, 2009. Disponível em: <http://periodicos.uefs.br/index.php/SSCF/article/view/SSCF-v.4-A1/4391>. Acesso em: 02 ago. 2020.

CORREIA, J.J.; MAGALHÃES, L. D. R. Obstáculos Epistemológicos na transposição didática do Calor. In: IX CÓLÓQUIO DO MUSEU PEDAGÓGICO. (ISSN 2175-5493), Vitória da Conquista (BA), Anais. Vitória da Conquista. MUSEU PEDAGÓGICO CASA PADRE PALMEIRA. 2009. v. 8, n. 1. p. 727-740, 2009. Disponível em: <http://periodicos.uesb.br/index.php/cmp/article/viewFile/3574/3265>. Acesso em: 10 nov. 2019.

CORREIA, J.J; JOSÉ, W. D. O conceito de trabalho de uma força em livros didáticos, In:__IX COLÓQUIO DO MUSEU PEDAGÓGICO (ISSN 2175-5493), Vitória da Conquista (BA), Anais. Vitória da Conquista. MUSEU PEDAGÓGICO CASA PADRE PALMEIRA. 2011. v. 9, n. 1, p. 727-740, 2011. Disponível em: <http://periodicos.uesb.br/index.php/cmp/article/viewFile/2663/2330>. Acesso em: 10 nov. 2019.

CORREIA, J.J; JOSÉ, W. D. O conceito de entropia e as leis da termodinâmica em livros didáticos de física. In:__ X CÓLÓQUIO DO MUSEU PEDAGÓGICO (ISSN 2175-5493), vol. 10, No 1, 2013. Vitória da Conquista (BA). MUSEU PEDAGÓGICO CASA PADRE PALMEIRA. 2013. Anais. Vitória da Conquista Disponível em: <http://periodicos.uesb.br/index.php/cmp/article/viewFile/3008/2717>. Acesso em: 10 nov. 2019.

CORREIA, J. J.; ORTIGOZA, L. V. O conceito de conservação de energia em livros didáticos: uma análise histórico-didática. Revista Binacional Brasil-Argentina: Diálogo entre as ciências, [S.1.], v. 4, n. 2, p. 91-103, set. 2015. ISSN 2316-1205. Disponível em: <http://periodicos2.uesb.br/index.php/rbba/article/view/1472>. Acesso em: 10 nov. 2019.

CORREIA, J. J. Definições de temperatura em fontes didáticas. Revista Binacional BrasilArgentina: Diálogo entre as ciências, [S.1.], v. 6, n. 1, p. 201-220, out. 2017. ISSN 2316-1205. Disponível em: <http://periodicos2.uesb.br/index.php/rbba/article/view/1520>. Acesso em: 10 nov. 2019. doi: https://doi.org/10.22481/rbba.v6i1.1520.

CORREIA, J. J.; OLIVEIRA, W. C. A Definição de Energia Interna e o enunciado da Primeira Lei da Termodinâmica nos livros didáticos. Revista Binacional Brasil-Argentina: Diálogo entre as ciências, [S.1.], v. 7, n. 2, p. 184-215, dez. 2018. ISSN 2316-1205. Disponível em: <http://periodicos2.uesb.br/index.php/rbba/article/view/4647>. Acesso em: 10 nov. 2019. 
A definição de 'entalpia' em livros didáticos. Revista Binacional Brasil-Argentina: Diálogo entre as ciências, [S.1.], v. 8, n. 1, p. 327-353, maio 2019. ISSN 2316-1205. Disponível em: <http://periodicos.uesb.br/index.php/rbba/article/view/4912>. Acesso em: Acesso em: 10 nov. 2019.

CORREIA, J. J; GUIMARÃES, G. F. Definição da energia livre de Helmholtz em fontes didáticas. Revista Binacional Brasil-Argentina: Diálogo entre as ciências, [S.1.], v. 8, n. 2, p. 141-169, mar. 2020. ISSN 2316-1205. Recuperado de: <https://periodicos2.uesb.br/index.php/rbba/article/view/7869>. Acesso em: 28 jan. 2021.

GILBERT, A. Origens Históricas da Física. Moderna: Introdução Abreviada. Fundação Calouste Gulbenkian. Lisboa. Portugal, 1982.

KONDEPUDI, D.; PRIGOGINE, I. Modern Thermodynamics: From Heat Engines to Dissipative Structures. Chichester: John Wiley \& Sons, 1998.

KORETSKY, M. Termodinâmica Para Engenharia Química. Rio de Janeiro: LTC, 2007.

LEITE, M. A. S. C. P.; ALMEIDA, M. J. B. M. Compreensão de termos científicos no discurso da ciência. Rev. Bras. Ensino Fís., São Paulo, v. 23, n. 4, p. 458-470, Dec. 2001. Disponível em:

$<$ http://www.scielo.br/scielo.php?script=sci_arttext\&pid=S180611172001000400011\&lng=en $\&$ nrm=isso $>$. Acesso em 12 out. 2019.

LEVINE, I. Físicoquímica, 5ª Edición, Volumen 1, Madrid: McGraw Hill, 2002.

MACEDO, H.; LUIZ, A. M. Problemas de Thermodinâmica Básica. Editora Edgard Blücher Ltda, 1978.

MORAN, M.; SHAPIRO, H.; BOETTNER, D.; BAILEY, M. Fundamentals of Engineering Thermodynamics, 8 a Edition, Nova Jersey: John Wiley \& Sons, 2014.

NAKAZA, R. M.; LINHARES, O. B. "O silogismo científico em Aristóteles" Jornada de Iniciação Científica e Mostra de Iniciação Tecnológica - ISSN 2526-4699 (2017). Disponível em https://philarchive.org/archive/NAKOSC. Acesso em 25 jun. 2020.

OLIVEIRA, M. J. Termodinâmica. São Paulo: Editora Livraria da Física, 2005.

PÁDUA, A. B.; PÁDUA, C. G. Termodinâmica - Uma Coletânea de Problemas. São Paulo: Editora Livraria da Física, 2006.

PORTO, J.; SILVA, J. O Potencial Químico e seu Ensino. Redequim, v. 5, n. 1, p. 211-224. Disponível em: <http://journals.ufrpe.br/index.php/REDEQUIM/article/view/2314/ 482483198>. Acesso em: 2 jun. 2020.

SEARS, F. W. e SALINGER, G. L. Termodinâmica, Teoria Cinética e Termodinâmica Estatística. $3^{\text {a }}$ Edição, Rio de Janeiro: Editora Guanabara Dois S.A, 1979. 


\section{A DEFINIÇÃO DE POTENCIAL QUÍMICO EM FONTES DIDÁTICAS}

SEARS, F. W. Física I - Mecânica, Movimento Vibratório e Calor. $3^{\text {a }}$ Edição, Rio de Janeiro: Ao Livro Técnico, 1966.

SMITH, J. M., NESS, H. C., ABBOTT, M. M. Introdução à Termodinâmica da Engenharia Química. 7 ed. Rio de Janeiro: LTC, 2007.

TSAPARLIS, G; SEVIAN, H. Introduction: concepts of matter - complex to teach and difficult to learn, in Tsaparlis G. and Sevian H. (ed.), Concepts of matter in science education, Dordrecht: Springer, pp. 1-8. 2013.

VAN WYLEN, G.J., SONNTAG, R. J. Fundamentos da termodinâmica clássica. $4^{\mathrm{a}}$ ed. São Paulo. Editora Blucher, 1975.

VAN WYLEN, G. J.; SONNTAG, R. E.; BORGNAKKE, C. Fundamentos da termodinâmica clássica. 4. ed. Tradução de Euryale de Jesus Zerbini e Ricardo Santilli Ekman Simões. São Paulo: Editora Edgard Blücher, 1995.

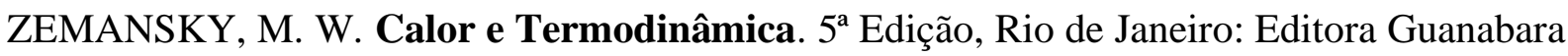
Dois S.A, 1978.

\section{NOTAS}

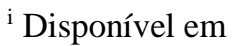
https://pt.wikipedia.org/wiki/Potencial_qu\%C3\%ADmico\#: :text=Quando\%20a\%20press\%C3\%A3o\%20\%C3 $\%$ A9\%20constante,energia\%20livre\%20est\%C3\%A1\%20no\%20m\%C3\%ADnimo. Acesso em: 1 jun. 2020.

ii Disponível em https://wikiciencias.casadasciencias.org/wiki/index.php/Potencial_Qu\%C3\%ADmico,_Fugacidade_e_Atividade. Acesso em: 1 jun. 2020.

iii Disponível em https://www.cesadufs.com.br/ORBI/public/uploadCatalago/11505901032012Fundamentos_de_FisicoQuimica_aula_8.pdf. Acesso em: 1 jun. 2020.
} 